\title{
Prevalence Of Urinary Tract Infection In Febrile Children Less Than Five Years Of Age
}

\author{
G Somaiah $^{1 *}$, Ashraf Mohinuddin Siddique' ${ }^{1}$, Suaraj Gupte ${ }^{1}$, Sai kiran ${ }^{1}$, B Raj Kumar ${ }^{2}$, P Jaya Ram ${ }^{2}$ \\ ${ }^{1}$ Department of Pediatrics, Mamata Medical College, Khammam, Andhra Pradesh. \\ ${ }^{2}$ Department of General Medicine, Mamata Medical College, Khammam, Andhra Pradesh.
}

\section{Abstract}

The aim of the present study was to diagnose the urinary tract infection in febrile children and to assess the validity of microscopic urine analysis and urine culture in the diagnosis of UTI. It is a prospective study conducted in a tertiary care hospital. 370 children were included in the study, data related to age, sex, nutritional status, socioeconomic status and predisposing risk factors like urethral instrumentation, bowel habits etc, were noted. A thorough physical examination with relevant investigations was carried out in all patients. Routine blood counts, urine analysis was done and those showing pus cells $>5$ per HPF in centrifuged urine sample were taken as study group and urine culture sensitivity was done in them.

A total of 370 febrile children were included in the study, out of 370 patients in study 165 were males and 205 were females with M: F ratio 1:1.2 and majority of them i.e. $64.8 \%$ were $<2$ years. In our study out of 370 children 48 children showed significant pyuria(12.9\%) of pyuric cases $26 \%$ showed significant bacterial growth making an overall prevalence of $3.5 \%$.Among culture positive UTI'S $76 \%$ were $<2$ years of age with a overall prevalence of $4.1 \%$ in children $<2$ years and $7 \%$ in children $<1$ year.

Key Words: urinary tract infection, febrile,children.

\section{INTRODUCTION}

Children with fever comprise a substantial
proportion of the practice in out patient department and Emergency Medicine. Fever is the most common reason for children under 5 years of age to visit Emergency/outpatient departments. Unlike occult bacteraemia or severe bacterial illness (in infants and children) little attention has been focused on the identification of urinary tract infections in febrile children in the emergency department, despite recent information that suggests a high prevalence of urinary tract infections and significant associated morbidity in these patients.

Fever and significant bacteriuria and pyuria in children with undocumented sources of infections must be presumed to be symptoms of pyelonephritis an invasive infection of the renal parenchyma requiring prompt treatment. It is essential to identify urinary tract infections in febrile children and institute prompt treatment to reduce the potential for life long morbidity. Progressive renal damage from unrecognized Pyelonephritis in childhood may lead to hypertension and chronic renal failure in later life. Urinary tract infection is defined as growth of a significant number of organisms of a single species in the urine, in the presence of symptoms. Significant bacteriuria is growth with a colony count of $>105 / \mathrm{ml}$ of a single species in a mid - stream clean catch urine sample. Most infections are caused by facultative anaerobes that usually originate from the flora of the bowel. Other pathogens such as group B streptococcus, staphylococcus epidermidis and candida albicans, originate in the flora of the vagina or perineal skin in women.

Byran CS et al (1984) [1] reported, Escherichia coli as the most common urinary pathogen accounting for $85 \%$ of community acquired urinary tract infection. Most UTIs are caused by uropathogenic Escherichia coli (UPEC) [2]. A

\section{Address for correspondence* \\ Dr. Somaiah \\ Department of Pediatrics,Mamata Medical College \& General Hospital,Khammam,Andhra Pradesh \\ Email: drsomaiahmmc@gmail.com}

retrospective UK study of 547 UTI cases (337 children aged $<16$ years) conducted in 2002-2008 found that 92\% of UTIs weere caused by E.coli. A prospective study in Brussels of 209 children (aged $<17$ years) with their first febrile UTI conducted in 20062008 found that the causative organism was E. coli in 91\% [3]. A retrospective study of 533 children (aged 6months to 6 years) conducted in 2001-2006 in the USA found that the causative organism was E. coli in $80 \%$ [4]. The problem with these studies is that urine sampling was not systematic. In addition, most included children were age dup to 16 or more. According to Arvind Bagga et al (2000) about $90 \%$ of first symptomatic urinary tract infection and $70 \%$ of recurrent infection's are due to Escherichia coli. Less commonly, other enteric gram negative bacteria such as Proteus or Klebsiella and Staphylococcus saprophyticus are responsible for community - acquired infections. The distribution of urinary pathogens in hospitalized patients is different, with E.coli accounting for about $50 \%$ of infections, whereas, proteus mirabilis is a common pathogen in males and in children with kidney stones and Klebsiella, Enterobacter, Citrobacter, Serratia, Pseudomonas aeruginosa, Providencia, Enterococcus and S.epidermidis accounting for most of the infections occur almost exclusively in hospitalized patients.

The present study was undertaken to determine the prevalence of urinary tract infection in febrile children, less than 5 years of age, and to assess the validity of microscopic urine analysis and urine culture in the diagnosis of urinary tract infection.

\section{MATERIALSAND METHODS}

The present study was conducted in the department of Pediatrics, Mamata Medical College and General Hospital, Khammam.

\section{Selection OfPatients}

Febrile children less than 5years attending the out patient department or admitted in the hospital over a period of 12 months were included in the study.

\section{Inclusion Criteria}

1. Febrile children between 1 month to 5years. 
2. Fever $\left\{\right.$ rectal $\geqslant 38.3^{\circ} \mathrm{C}$ or axillary temperature $\left.\geqslant 37.8^{\circ} \mathrm{C}\right\}$.

Exclusion Criteria

1. Children below 1 month and above 5 years.

2. Any child who has received antibiotics 48 hours prior were not be included in the study.

3. Children with known congenital genitourinary anomalies.

Methods Of Study

370 children were included in the study, data related to age, sex, nutritional status, socioeconomic status and predisposing risk factors like urethral instrumentation, bowel habits etc, were noted. A complete history related to the onset, duration of fever and associated symptoms such as nausea, vomiting, diarrhea, urinary disturbances, other system involvement was obtained.

A thorough physical examination with relevant investigations was carried out in all patients. Routine blood counts, urine analysis was done and those showing pus cells $>5$ per HPF in centrifuged urine sample were taken as study group and urine culture sensitivity was done in them, USG examination were done, in culture positive cases, one case DTPA scan was done, the detailed data was entered in the Proforma.

Collection Of Urine Sample

From all 370 cases a sample of urine was collected. In children under 2 years of age urine was collected by a bag and in others midstream sample was collected.

\section{Collection Of Bag Sample}

In children below 2 years of age the genitalia was cleaned with soap and water and person collecting sample washed hands before touching the bottle or bag for collecting urine sample. In males prepuce retracted if possible, in females below 2years labia was split apart and washed. Urine was collected in bag; around $10 \mathrm{ml}$ of urine was transferred into sterile bottle and sent for culture and sensitivity .In children above 2years midstream sample was collected.

\section{Method Of Collection Of Mid Stream Sample}

After taking the above precautions child was allowed to pass urine, mid stream sample was collected in sterile bottle and was sent for culture and sensitivity.

\section{Urine Analysis}

The fresh urine samples obtained from the above techniques were subjected for urinalysis and culture and sensitivity. The urine specimens were centrifuged in a standard manner, $10 \mathrm{ml}$ of urine was span at the rate of $2500 \mathrm{rpm}$ for 20-30mintes, supernatant decanted off and sediment resuspended in the remaining $0.2 \mathrm{ml}$. The urine was examined under microscope for Hematuria, and Leukocyturia.In the present study more than 5 pus cells/HPF in a centrifuged urine sample was taken as significant pyuria and culture and sensitivity was performed in that patient/ case.

\section{Urine Culture}

The clean catch mid stream urine was inoculated into blood and macconkey agar plates with a $0.01 \mathrm{ml}$ calibrated loop. All plates were incubated at $35-370 \mathrm{C}$ for $24 \mathrm{hrs}$ under aerobic condition to obtain accurate colony count. On culture of mid stream sample of urine, a colony count of more than $105 / \mathrm{ml}$ organisms of a single species was considered significant.

Samples showing insignificant growth, mixed growth of two or more pathogens or growth of non-pathogens were not considered as culture positive. The following definitions were employed in the present study.

Significant Pyuria sample.

Presence of more than 5 pus cells /HPF in a centrifuged urine Positive Urine Culture

A positive urine culture was defined as growth of $>105$ colonies of a single urinary tract pathogen $/ \mathrm{ml}$ of specimen in a mid stream of urine.

\section{RESULTS}

In the present study 48 children (12.9\%) showed Pyuria in centrifuged urine sample of which $26(54.16 \%)$ were males and $22(45.8 \%)$ were females. Majority were $<2$ years $58 \%$ (Table- 1 ).

In our study growth $>105 \mathrm{CFU} / \mathrm{ml}$ of single organism was considered significant growth. $73 \%$ of Pyuric children showed no significant growth on Urine culture. Among positive cultures $69.33 \%$ showed E.coli, $7.6 \%$ showed acinobacter, citrobacter, pseudomonas, and serratia species (Table-2).

\section{DISCUSSION}

Urinary tract infections are common, potentially serious infections of childhood. They cause acute morbidity as well as long term squeal including hypertension and impaired renal function. Accurate diagnosis of urinary tract infection is important to facilitate appropriate management of acute illness, and to ensure appropriate evaluation and follow up. Equally important is accurately ruling out a urinary tract infection to avoid unnecessary cost and potentially harmful treatment and evaluation.

The present study was a prospective study conducted in department of pediatrics, Mamata Medical College and General Hospital over a period of 12 months to determine the magnitude of urinary tract infection in febrile children between 1month to

Table-1: Age and Sex distribution of subjects with urine showing $>5$ pus cells/ HPF

\begin{tabular}{|c||c|c|c||}
\hline \multirow{2}{*}{ Age } & \multicolumn{2}{|c||}{ Sex } & \multirow{2}{*}{ Total } \\
\cline { 2 - 3 } & Male & Female & 16(33.33) \\
\hline \hline < year & $6(23.07)$ & $10(45.45)$ & $12(25.00)$ \\
\hline \hline 1-2 years & $8(30.77)$ & $4(18.18)$ & $120.42)$ \\
\hline \hline 2-3 years & $4(15.38)$ & $1(4.55)$ & $5(10.42)$ \\
\hline 3-4 years & $5(19.23)$ & $4(18.18)$ & $9(18.75)$ \\
\hline 4-5 years & $3(11.54)$ & $3(13.64)$ & $6(12.50)$ \\
\hline \hline Total & $26(100.0)$ & $22(100.00)$ & $48(100.00)$ \\
\hline \hline
\end{tabular}

Table-2: Distribution of Urine Culture

\begin{tabular}{|c|c|c|c|}
\hline \multirow{2}{*}{ Urine culture report } & \multicolumn{2}{|c|}{ Sex } & Total \\
\cline { 2 - 4 } & Male & Female & \\
\hline No growth & $18(69.2)$ & $17(77.27)$ & $35(72.91)$ \\
\hline E.coli & $5(19.23)$ & $4(18.18)$ & $9(18.75)$ \\
\hline Acinobacter & ---- & $1(4.55)$ & $1(2.08)$ \\
\hline Citrobacter & $1(3.85)$ & ---- & $1(2.08)$ \\
\hline Pseudomonas & $1(3.85)$ & ---- & $1(2.08)$ \\
\hline Serratia species & $1(3.85)$ & ---- & $1(2.08)$ \\
\hline Total & $26(100.00)$ & $22(100.00)$ & $48(100.0)$ \\
\hline
\end{tabular}


5years and also to assess the validity of routine microscopic urine analysis and culture in the diagnosis of urinary tract infection. A total of 370 febrile children were included in the study, out of 370 patients in study 165 were males and 205 were females with M:F ratio 1:1.2 and majority of them i.e.64.8\%were $<2$ years.

In our study out of 370 children 48 children showed significant pyuria $(12.9 \%)$ of pyuric cases $26 \%$ showed significant bacterial growth making an overall prevalence of $3.5 \%$.Among culture positive UTI'S $76 \%$ were $<2$ years of age with a overall prevalence of $4.1 \%$ in children $<2$ years and $7 \%$ in children $<1$ year.

Prevalence of febrile UTI in infants in our study is almost similar to study by Dharni Dharaka et al (1993) [5] who reported a prevalence of $5.4 \%$ in febrile infants, Hoberman et al (1993) [6] who reported prevalence of 5.3\% in infants.

Overall prevalence of UTI in febrile children in our study was $3.5 \%$ and $7 \%$ in children $<5$ years and infants respectively in contrast to study conducted by Kaushal et al (2003) [7] who reported higher prevalence of $8.4 \%$ and $12.3 \%$ in children $<5$ years and infants respectively.

Overall prevalence of febrile UTI in infants in our study (7\%) was higher compared to report by Shaw K.N et al (1998) from USA who reported prevalence of $3.3 \%$ in febrile infants. In our study prevalence of UTI in <2years age group was $4.1 \%$ which was similar to study by Roberts et al (1983) [8] who coated prevalence of $4.1 \%$. M:F ratio of culture positive cases in the age group of $<2$ years was 1:1 and in children $>2$ years there was male preponderance. Although children with known renal anomalies were excluded in our study. We detected renal anomalies for the first time (6 out of 13) by USG examination, this explaining the male preponderance in our study.

Among culture positive cases $69 \%$ grew E. coli and $7 \%$ each of pseudomonas, citrobacter, acinobacter, serratia species, which correlates with other studies. Bryan et al (1984) [1] reported E. coli as the common urinary pathogen in $85 \%$ of cases. According to Aravind Bagga et al (2000)90\% of first symptomatic urinary tract infection and $70 \%$ reccurence infections were due to E.coli. Hoberman et al (1993) [6] reported as E.coli as the most common bacterium isolated in his study.

Because of economical constraints urine cultures were done only in children who showed significant pyuria which revealed positive culture in $26 \%$. Hence validity of urine examination could not be accurately ascertained. In our study $40 \%$ of children who showed numerous pus cells were culture positive and $44 \%$ who showed $>10 \%$ were culture positive and $2.5 \%$ of children showing $>5$ pus cells were culture positive. Hence the presence of pyuria of $>5$ leukocytes/HPF in a centrifuged sample is a significant indicator of UTI. Clinicians should be aware of the possibility that febrile children may have urinary tract infection and should consider obtaining a urine culture specimen as part of their diagnostic evaluation. The presence of another potential source of fever such as upper respiratory tract infection or otitis media is not reliable in excluding urinary tract infection. Several studies in developed countries have shown a low prevalence $(1.7-4.1 \%)$ of urinary tract infection in febrile children. Present study reveals similar overall prevalence of UTI (3.5\%) in febrile children 1 month to 5 years and $4.1 \%$ in children $<2$ years and $7 \%$ in children $<1$ year of age.

\section{CONCLUSION}

The study would have been more conclusive if urine cultures were done in all febrile children screened, but economical constraints limited us to do urine culture only in those children showing significant pyuria of $>5$ pus cells/ HPF of centrifuged urine sample and we found that $26 \%$ of febrile pyuric children were culture positive hence the validity of study cannot be assessed. Prevalence of culture positivity was $44 \%$ in those who showed $>10$ pus cells/HPF in centrifuged sample of urine compared to $2.5 \%$ in those who showed $>5$ pus cells/HPF. Hence we conclude that pyuria of $>5$ pus cells /HPF in centrifuged sample should be considered as significant pyuria and further evaluation should be done promptly to initiate treatment and to prevent morbidity and long term sequele.

REFERENCES1. Bryan CS, Reynolds KL. Community acquired Bacteremic urinal; tract infection: Epidemiology and Outcome. J Urol 1984; 132: 490, 934.

2. Hannan TJ, Totsika M, Mansfield KJ, Moore KH, Schembri MA, HultgreSJ. Host-pathogen checkpoints and population bottlenecks in persistent and intracellular uropathogenic. Microbiology Reviews 2012;36(3):616-48.

3. Ismaili K, Wissing KM, Lolin K, Le PQ, Christophe C, Lepage $\mathrm{P}$, et al. Characteristics of First Urinary Tract Infection With Fever in Children. A Prospective Clinical and Imaging Study. The Pediatric Infectious Disease Journal 2011;30(5):371-74.

4. Paschke AA, Zaoutis T, Conway PH, Xie D, Keren R. Previous Antimicrobial Exposure Is Associated With Drug-Resistant Urinary Tract Infections in Children. Pediatrics 2010;125:664-72.

5. Dharnidharka VR. Prevalence of bacteriuria in febrile infants. Indian Pediatr 1993; 30: 981-986.

6. Hoberman A, Chao HP, Keller DM, Hickey R, Davis HW, Ellis D. Prevalence of uninary tract infection in febrile infants. J pediatr 1993; $123: 17-23$.

7. Kaushal R.K, Bansal S, Sharma V.K, Sood A, Goyal A Urinary tract infection among children presenting with fever. Indian Pediatr 2003; 40:269-270.

8. Roberts KB, Charney E, Sweren RJ, Ahonkhai VI, Bergman DA, coulter MP et al: Urinary tract infection in infants with unexplained fever: a collaberative study. J Pediatr 1983; 103: 864-867. 